Para enlazar con este artículo / To link to this article:

http://dx.doi.org/10.14198/fem.2018.32.09

Para citar este artículo / To cite this article:

Pérez del Hoyo, Raquel. «Integración de la perspectiva de género en el estudio de la ciudad y su patrimonio: aprendiendo de la experiencia de Benalúa hacia un futuro más sostenible». En Feminismo/s, 32 (diciembre 2018): 231-257. Dosier monográfico: MAS-MES: Mujeres, Arquitectura y Sostenibilidad - Medioambiental, Económica y Social, coord. María-Elia Gutiérrez-Mozo, DOI: 10.14198/fem.2018.32.09

\title{
INTEGRACIÓN DE LA PERSPECTIVA DE GÉNERO EN EL ESTUDIO DE LA CIUDAD Y \\ SU PATRIMONIO: APRENDIENDO DE LA EXPERIENCIA DE BENALÚA HACIA UN FUTURO MÁS SOSTENIBLE
}

\author{
GENDER MAINSTREAMING INTO THE STUDY \\ OF THE CITY AND ITS HERITAGE: LEARNING \\ FROM THE EXPERIENCE OF BENALÚA TOWARDS \\ A MORE SUSTAINABLE FUTURE
}

\author{
Raquel PÉREZ DEL HOYO \\ Universidad de Alicante \\ perezdelhoyo@ua.es \\ orcid.org/0000-0002-0104-6153
}

\section{Resumen}

En este artículo investigamos la importancia de incorporar la perspectiva de género a los indicadores de valoración del patrimonio urbano y arquitectónico a través del estudio del barrio de Benalúa de Alicante en España. Para ello, desarrollamos un análisis tridimensional del barrio, su arquitectura y habitantes, abordando los ámbitos de: 1) la planificación urbana, 2) el diseño de la casa y 3) la actividad ciudadana. El papel activo que desempeñan las mujeres en la propuesta del modelo de Benalúa, un barrio inclusivo construido por una comunidad diversa, abre un campo de reflexión que nos permite aprender y avanzar hacia modelos de ciudades más sostenibles. De la investigación se concluye que valores tales como los espacios públicos de relación, los equipamientos y servicios, la movilidad y accesibilidad, las viviendas, la seguridad y la participación ciudadana, son variables pertinentes no sólo en el análisis y en el 
Integración de la perspectiva de género en el estudio de la ciudad y su patrimonio: aprendiendo de la experiencia de Benalúa hacia un futuro más sostenible

proyecto de la ciudad y su arquitectura, sino también y muy especialmente en su puesta en valor como patrimonio sostenible.

Palabras clave: perspectiva de género, sostenibilidad, urbanismo, arquitectura, patrimonio.

\section{abstract}

This article investigates the importance of gender mainstreaming into the indicators of urban and architectural heritage valuation through the study of the Benalúa neighbourhood of Alicante in Spain. To this end, it is developed a three-dimensional analysis of the neighbourhood, its architecture and inhabitants, addressing the areas of 1) urban planning, 2) housing design and 3) civic activity. The active role played by women in the proposal of the Benalúa model, an inclusive neighbourhood built by a diverse community, opens a field of reflection that allows us to learn and move towards more sustainable models of cities. The research concludes that values such as public spaces for relationships, facilities and services, mobility and accessibility, housing, security and citizen participation are relevant variables not only in the analysis and design of the city and its architecture, but also and especially in its enhancement as a sustainable heritage.

Keywords: Gender mainstreaming, sustainability, urban planning, architecture, heritage.

\section{INTRODUCCIÓN Y OBJETIVOS}

La irrupción del nuevo escenario que nos plantea la Agenda 2030 para el Desarrollo Sostenible, aprobada en el año 2015 en la Asamblea General de las Naciones Unidas, nos invita a reflexionar sobre la sostenibilidad del actual modelo de desarrollo y la necesidad de crear uno nuevo mediante la consecución de los Objetivos de Desarrollo Sostenible (ODS). Entre los ODS se incluye el 11: «Lograr que las ciudades y los asentamientos humanos sean inclusivos, seguros, resilientes y sostenibles» (ONU 16).

Para apoyar el logro de este objetivo, las metodologías basadas en la investigación de casos singulares de éxito, con el objeto de identificar y aprender de las buenas prácticas, resultan muy adecuadas. Como indica Hernández Aja, para que una actuación urbana pueda calificarse de Buena Práctica, al menos debe reunir los siguientes requisitos:

Feminismo/s 32, diciembre 2018, pp. 231-257 
Integración de la perspectiva de género en el estudio de la ciudad y su patrimonio: aprendiendo de la experiencia de Benalúa hacia un futuro más sostenible

Tener un impacto demostrable y tangible en la mejora de las condiciones de vida de las personas.

Ser el resultado del trabajo conjunto entre los diferentes sectores que actúan y viven en la ciudad: la administración, los ciudadanos a través de sus asociaciones y el sector privado en general.

Ser social, cultural, económica y ambientalmente sostenibles y duradera.

Contribuir al fortalecimiento de la comunidad y de su capacidad de organización.

Prestar especial atención a la resolución de los problemas de exclusión social ya sea de género, cultural, étnica o económica. (Hernández Aja)

Reconocidos estudios constatan la relevancia del nacimiento del barrio de Benalúa de Alicante (1884), iniciativa adelantada a su tiempo y lugar que, anticipándose incluso a la ejecución del Ensanche de la ciudad (1898), apostó por reformular, desde las bases mismas de la cultura moderna, el complejo concepto de ciudad, sus modos de vida y relaciones humanas, sus valores técnicos, funcionales y estéticos. De este modo, Benalúa se muestra sugerente e inagotable, reclamando nuevas visiones actuales capaces de desvelar su identidad, razón y cualidad de ser, invitando a abordar estudios desde las perspectivas más innovadoras e integradoras, como la que el género aporta.

En este sentido, el presente artículo muestra los resultados de las investigaciones realizadas en la Universidad de Alicante, orientadas a reconsiderar Benalúa desde la perspectiva de género, apasionante iniciativa que nos permitirá redescubrir un auténtico proyecto moderno, el cual, más allá de revelarse en su particular contexto, último tercio del siglo XIX en una ciudad española de provincias, cobra si cabe mayor importancia en el crítico momento actual, ante el reto de definir un nuevo modelo de desarrollo sostenible, manteniendo su valía e incluso primacía frente a ciertas actuaciones contemporáneas.

Con especial interés, se discuten los temas que conciernen a la perspectiva de género en la planificación urbana: el espacio público, la movilidad, los equipamientos para la vida cotidiana, la seguridad, el hábitat y la participación ciudadana. En Benalúa, por ejemplo, los espacios públicos están ordenados y son homogéneos, perfectamente acotados conforme a la ideología higienista sobre la base en retícula de manzanas seriadas ajustadas a la escala humana parcelaria, unidades válidas tanto para la construcción de viviendas como de equipamientos o plazas, eliminando jerarquías de otras épocas para permitir

Feminismo/s 32, diciembre 2018, pp. 231-257 
en igualdad y con total flexibilidad el desarrollo de las diversas funciones y roles sociales, públicos o privados, de mujeres y hombres.

Se debaten también los aspectos que afectan al diseño de la casa, concebida en dos niveles diferenciados, con amplitud suficiente para promover empresas familiares, servicios profesionales o actividades comerciales como extensión de la propia vivienda y de la propia vida, interviniendo las familias activamente en la configuración del barrio, al tiempo que surge la pequeña ciudad debiendo equiparse y autoabastecerse, iniciativas en las que las mujeres, especialmente, encontrarán la oportunidad de incorporarse al mundo productivo frente al tradicional reproductivo.

Se reflexiona asimismo sobre la intensa actividad social y cultural desarrollada en el barrio, escogido como residencia por muchos de los ilustres personajes de la época, circunstancia que, sin duda, confirió a Benalúa un valor adicional consolidando su singular modernidad. No de forma casual, el amor por la cultura y las manifestaciones artísticas acompañó, por un lado, la idea de defender la integración de diferentes grupos sociales (profesionales, empresarios, comerciantes o trabajadores) erigiéndose el barrio como ejemplo de convivencia y de puesta en valor de la diferencia; y, por otro, la convicción y el empeño en la necesidad prioritaria de procurar la educación a todas las niñas y a todos los niños sin distinción, posibilitando a las mujeres adquirir en igualdad la formación que abriría las puertas a sus aspiraciones personales y laborales.

Por ello, debe considerarse la perspectiva de género como una categoría de análisis decisiva e identificativa de la modernidad del barrio, sumándose al interés de los aspectos urbanísticos, higiénicos, sociales, culturales y de industrialización, que convierten en singular y adelantado el proyecto de Benalúa. Sin duda, una buena práctica a considerar en la definición de un nuevo modelo urbano de desarrollo más inclusivo, seguro, resiliente y sostenible, capaz de afrontar los retos de las ciudades del siglo XXI.

Pero la perspectiva de género no sólo nos aporta una visión clave para entender en su compleja totalidad el barrio, sino que también y además se erige, y así se reivindica en el presente artículo, como un parámetro crucial, y hasta ahora obviado, en la valoración patrimonial de su arquitectura y de su urbanismo. El legado de la modernidad (y en este escrito entendemos por tal lo ocurrido desde la triple revolución, política, industrial y cultural, de

Feminismo/s 32, diciembre 2018, pp. 231-257 
mediados del XVIII hasta la actualidad) presenta, en estas disciplinas, características peculiares que hemos de reconsiderar para abordar su conservación, puesta en valor y actualización.

Frente a posiciones arqueológicas o, en el diámetro opuesto, de hacer tabla rasa, la perspectiva de género desvela el valor de la vida que palpita bajo estos proyectos y cuyo entendimiento cabal nos da las pistas para su respeto y aprecio.

\section{DEL NACIMIENTO DE BENALÚA Y LA IGUALDAD DE OPORTUNIDADES ENTRE MUJERES Y HOMBRES}

Claro es, y la justicia exige que se reconozca, que la mujer con su labor perseverante se ha abierto camino, ha derribado esas barreras que parecían infranqueables y ha demostrado que puede y sabe tomar parte en la vida de lucha social que nos rodea (Echarri 434).

En el último tercio del siglo XIX, la actuación de Benalúa, un barrio de casas en las afueras de la capital, supuso duplicar (desdoblar) Alicante, abarcando una extensión superficial casi equivalente a la mitad de la entonces ocupada por el casco urbano (Fig. 1). Alejado ochocientos metros hacia el oeste de la población, en contacto con el campo, el barrio de Benalúa, pensado y planificado conforme a los criterios higienistas y urbanísticos más avanzados del momento (Alonso Salvador, Blasco Sánchez y Piñón Pallarés 66-69), nació con voluntad de erigirse como modelo suburbano de ciudad nueva, bien comunicado, autosuficiente, sin herencias cuestionables, accesible en ausencia de jerarquías arrastradas de otras épocas: diferencias de género, estatus o procedencia. Así, puede afirmarse que Benalúa formó parte de las primeras propuestas originarias del urbanismo que abordaron de forma holística los problemas de la ciudad, su relación con el campo y la movilidad, apostando por el progreso y la armonía social. Un enfoque clave en el momento actual que refuerza el paradigma ecológico en su idea fundamental: que la solución a los problemas sólo puede provenir del equilibrio entre las acciones desarrolladas en diferentes ámbitos y con diferentes perspectivas (Verdaguer VianaCárdenas, «El campo y la ciudad»). ¿Cómo fue posible, en su momento y lugar, llevar a cabo una obra de tal magnitud y concepción moderna?

Feminismo/s 32, diciembre 2018, pp. 231-257 
Integración de la perspectiva de género en el estudio de la ciudad y su patrimonio: aprendiendo de la experiencia de Benalúa hacia un futuro más sostenible

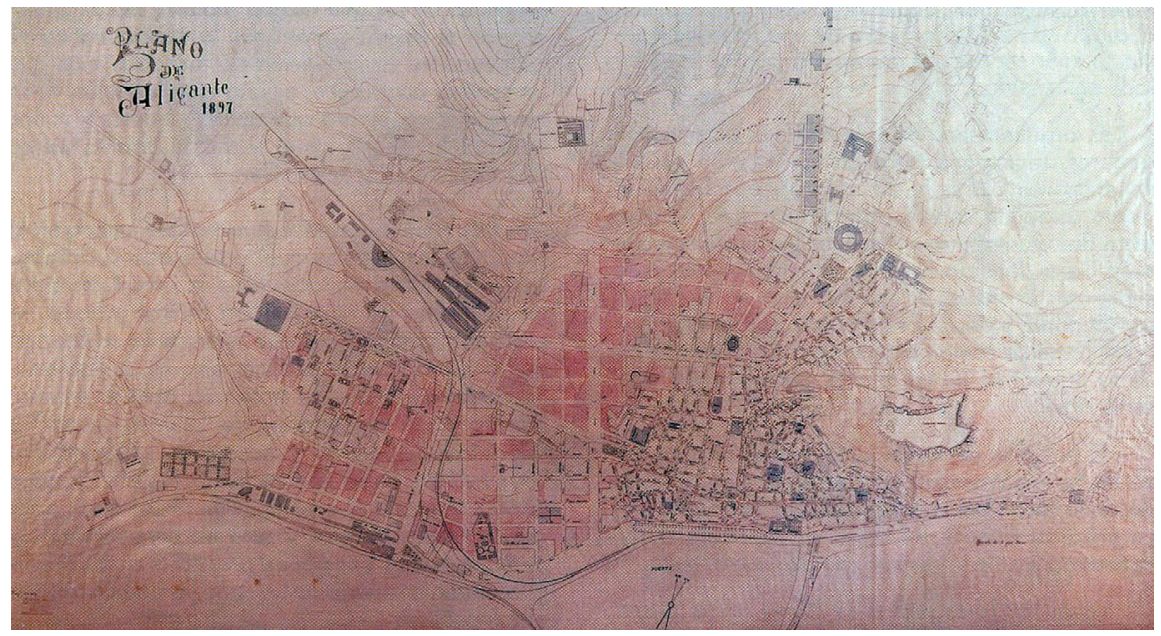

Figura 1. «Plano de Alicante 1897». Biblioteca Gabriel Miró. Fuente: (Bevià i Garcia y Varela Botella 106).

Cuando se concibió la idea de construir Benalúa, por iniciativa particular de la Sociedad Anónima Los Diez Amigos, el casco urbano alicantino soportaba una densidad aproximada de ciento setenta viviendas por hectárea, creciendo la población al ritmo que prosperaba el comercio portuario (Gutiérrez Lloret 541-560). La escasez de viviendas, sobre todo económicas, generó un verdadero problema, sumándose a las carencias de agua e higiene que, desde mediados de siglo, venían provocando sucesivas epidemias de cólera y fiebre amarilla (Ponce Herrero y Dávila Linares 141-159). Mientras tanto, el Ayuntamiento de Alicante, sin hacer frente a estas extremas y dramáticas circunstancias que directamente afectaban a la mujer por considerarse en la sociedad (puertas adentro) responsable del funcionamiento del hogar y del bienestar de la familia, prácticamente había paralizado las gestiones para iniciar la construcción de ensanches que ampliaran la ciudad.

Al margen de cualquier interés especulativo (que queda fuera del objeto de este estudio), fueron sin duda estas dos razones las que propiciaron el nacimiento de Benalúa, a pesar de no existir en Alicante un marco normativo que regulara este tipo de propuestas. Por un lado, atender la demanda social del momento, ofreciendo espacios saludables y viviendas higiénicas para

Feminismo/s 32, diciembre 2018, pp. 231-257 
habitar (proponiendo una densidad aproximada de tan sólo ochenta y ocho viviendas por hectárea). Y, por otro, suplir la ineficaz gestión municipal de la época, incapaz de sacar adelante un proyecto oficial de ensanche.

De este modo, Benalúa parte de cero, como un sueño, sin lastres, con total libertad. Es la oportunidad y el proyecto común de promotores y familias, futuras propietarias de una casa, basado en la unión de esfuerzos y voluntades. Una ambiciosa empresa, precursora de la vivienda social (Miralles 8), concebida al margen de directrices o estrategias municipales impuestas, capaz de autogestionarse y autofinanciarse. De hecho, funcionando a modo de cooperativa, contó exclusivamente para costear su ejecución con los importes por la emisión de doscientas acciones que significaban doscientas futuras casas, adquiridas mediante cuotas mensuales por familias de diversa procedencia y clase social; y con los ingresos por la compraventa de terrenos destinados directamente a la construcción particular.

Así, con especial sensibilidad y relevancia, cuando en España resultaba evidente la discriminación de la mujer (Código Penal de 1870) y se gestaba legalmente, aún más si cabía, la desigualdad de género en el país (Códigos de Comercio de 1885 y Civil de 1889), Benalúa representó la ilusión compartida de mujeres y hombres. En igualdad de oportunidades, Teresa Espí Oltra fue la primera mujer en adquirir terrenos en el barrio, siguiendo su iniciativa Ángela Baldó Compañ, Rafaela Juan Urios, Rosario Beltrán Torregrosa, Eulalia Picó Martínez y Teresa Urios Lacárcel. Asimismo, con nombres y apellidos (que no deben obviarse: lo que no se nombra, no existe), figuraron como accionistas: la misma Teresa Espí Oltra, Teresa Picó López, Rosario Hernández de Tejada, Valentina de Aguilera, Francisca Sánchez Rubio, Dolores Pascual Torrent, María de la Concepción Álvarez y Belluga, Inocencia Beltrán, Dolores Sánchez Such, Nieves Pastor de Terol, Josefa García Sereix, Carmen Pastor, Concepción Pro Sanmartín, Josefa Avela Manzanaro, Josefa Pérez Urios, Rafaela Pastor Esplá, Piedad Guardiola Forgas, Flora Guardiola Boix, Dolores Sirvent Manero, Rafaela Tonda Gómez y María Luisa Lledó Mallol (Pardo Jimeno). En total, pueden documentarse, desde el inicio de la construcción del barrio, las identidades de veinticinco mujeres accionistas o propietarias en el moderno Benalúa, pionero en apostar por su natural integración en ejemplar convivencia.

Feminismo/s 32, diciembre 2018, pp. 231-257 
La participación de la mujer (mayor del diez por ciento), empresaria y emprendedora, en el proyecto sostenible (todavía idea o sueño) de Benalúa, paladín de los derechos (entonces soslayados) que por naturaleza le correspondían (Nash), supone un dato (hecho) inquietante de partida, tan apasionante como incomprensiblemente ausente en las investigaciones en la materia (hasta el momento, de autoría mayoritariamente masculina), suficiente para motivar el estudio en profundidad de este barrio, en el sentido de contribuir a subsanar las carencias manifiestas de su conocimiento. Porque resulta evidente que, sin la perspectiva actual e innovadora que el género aporta, como argumenta este artículo, no puede concebirse cabalmente el entendimiento de la experiencia y enseñanzas de Benalúa.

\section{BENALÚA: PRIMERA PLANIFICACIÓN MODERNA DE ALICANTE Y PROYECTO DE VIDA SOSTENIBLE}

Tiene Benalúa muchos encantos: el de su nacimiento, el del lugar donde radica, el de su pequeña historia. Nace geométricamente en bloque: sus primeros habitantes-propietarios se distinguen por su sentido de hermandad; su situación entre lugares bien determinados, tan concretos, la hacen única en Alicante.

...tiene su propia vida impregnada de algo bello y que no es fácil describir... Enclavada en la ciudad, viviendo «en ella» sin embargo, tiene las características propias como si fuera una sola unidad (Miralles 8).

El proyecto de Benalúa, firmado por el arquitecto José Guardiola Picó con fecha 20 de octubre de 1883, forma parte del conjunto de actuaciones que, por primera vez en España, en la transición de los siglos XIX al XX, trataron de concebir, racionalizar y controlar el crecimiento urbano, proponiendo pensar y planificar la ciudad en su globalidad. Si bien esta función correspondía a los ensanches generales de las ciudades, como hemos introducido, en el caso de Alicante (por su demora), Benalúa se anticipó, asumiendo esta responsabilidad de forma sectorial y por iniciativa particular.

Resultaba evidente que, con el paso del tiempo, el barrio quedaría incluido en el perímetro del ensanche de la población, de ahí la toma de ciertas decisiones proyectuales en su materialización: sección de calles o definición de chaflanes; aunque no deben confundirse estos aspectos formales con el 
verdadero origen e idiosincrasia del barrio. Benalúa nunca se pensó como extensión de Alicante, surgió precisamente por significar y representar los valores contrarios y, por tanto, separada como ciudad independiente, alejada de los problemas y prejuicios adquiridos del casco urbano tradicional, erigiéndose como primera planificación moderna de Alicante (Giménez García, Giner Álvarez y Varela Botella 26-30).

De hecho, uno de los valores más relevantes del trazado de Benalúa corresponde a su escala, a su dimensión abarcable, acotada y precisa. Sin tener que responder estrictamente a las dimensiones del ensanche, sin imposiciones, la nueva ciudad moderna se humaniza en absoluta armonía geométrica. De este modo, Benalúa se proyecta a escala de barrio, permitiendo al habitante su control. El orden de la trama facilita, además, la comprensión de los diversos espacios que componen la planificación (construidos o vacíos, públicos o privados) y, en consecuencia, la apropiación del conjunto proyectado (Fig. 2). Así, el habitante desarrolla el necesario sentimiento de pertenencia, conoce y se identifica con un determinado tejido urbano y con cada uno de sus espacios componentes, los cuales, al integrarse en la matriz, percibe propios, complementarios y en equilibrio.

La ciudad que así se concibe, manifiesta la voluntad por mostrarse accesible, amable, cercana a las necesidades individuales y comunitarias, sin generar dependencias que limiten la movilidad o el desarrollo de la vida cotidiana. Se descubre, por tanto, y especialmente, a favor del género y las clases sociales más desfavorecidas, funcionando como espacio integrador y base conciliadora de las relaciones humanas. Se hace posible, de este modo, un urbanismo feminista para la vida cotidiana, definiendo un claro estilo de vida a favor de la integración (Col-lectiu Punt 6). El habitante, sin distinción, porque comprende y le es propio, percibe alcanzable un entorno sugerente, seguro, sin barreras ni obstáculos añadidos que mermen su confianza y, en la medida en que lo necesita, dispone del mismo en equidad. No es más que el reflejo de una sociedad que avanza enriqueciéndose, que madura en el empeño de mejorar constantemente su espacio físico más cercano, la ciudad que habita, la ciudad de todos. De este modo se concibe y desarrolla Benalúa. Por encima de roles sociales asignados (no asumidos), mujeres y hombres, niñas y niños, ancianas y ancianos, de diversa procedencia o estatus, acceden (conviven) en igualdad y valoran su diferencia. Especialmente, por su particular devenir

Feminismo/s 32, diciembre 2018, pp. 231-257 
Integración de la perspectiva de género en el estudio de la ciudad y su patrimonio: aprendiendo de la experiencia de Benalúa hacia un futuro más sostenible

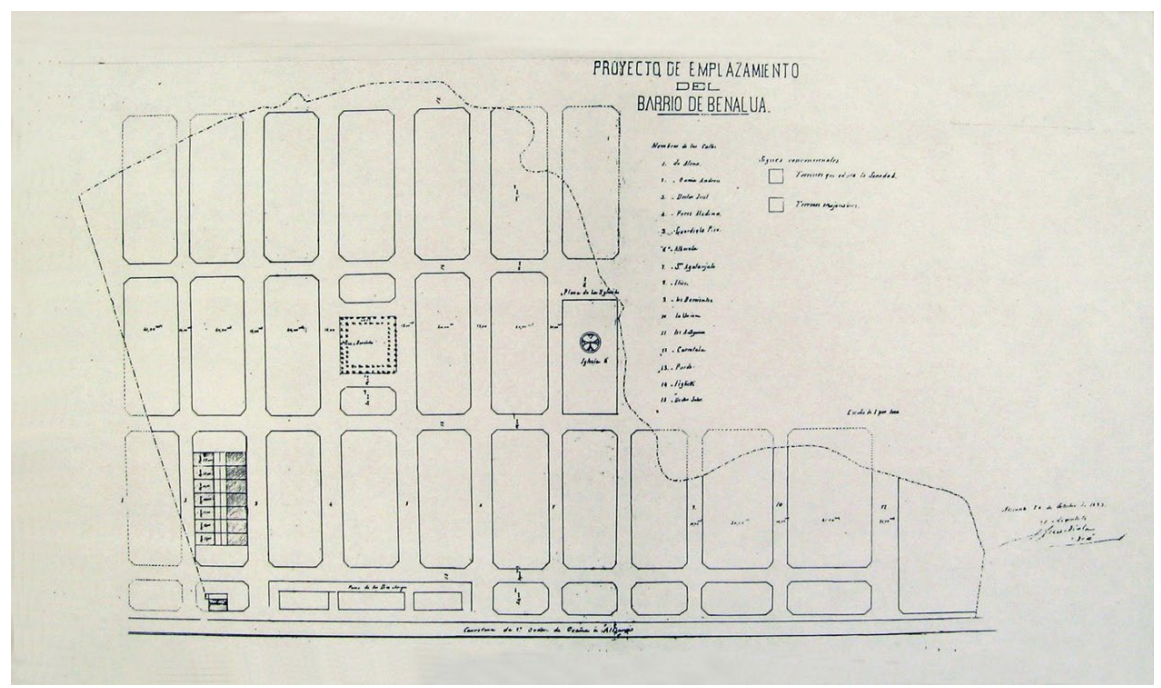

Figura 2. Plano de planta general del proyecto de Benalúa (arquitecto Guardiola Picó, 1883). Fuente: Archivo Histórico Municipal de Alicante.

histórico, en Benalúa la mujer (igual que el hombre) controla y comprende un hábitat que siente propio más allá de la intimidad del hogar, porque puede valerse por sí misma toma conciencia de su importancia individual, sólidos cimientos sobre los que alzar la voz y, por qué no, sus más ambiciosos proyectos y aspiraciones personales. Es el propio espacio urbano de Benalúa el que custodia las reglas que, como afirma Isabela Velázquez, corresponden exactamente a la estructura de poderes y relaciones que rigen la sociedad del barrio.

Así, más allá de resolver las cuestiones meramente técnicas, Benalúa propone un proyecto de vida sostenible, de sociedad moderna, participativa y responsable, consciente de la necesidad de mantener el valor de un hábitat propio que trasciende el perímetro de la casa, y en este sentido avanza. Benalúa es un proyecto de ciudad al servicio de un proyecto de vida: por eso la figura del arquitecto cede protagonismo a la del ciudadano y habitante, que llevan la voz cantante. Es la implicación de la ciudadanía en la definición de los temas que requieren solución, así como de los criterios y prioridades de actuación, la que conduce el proceso de formación del barrio hacia

Feminismo/s 32, diciembre 2018, pp. 231-257 
un urbanismo sostenible (Hernández Aja, Velázquez Valoria y Verdaguer Viana-Cárdenas).

Cuando comienza el siglo XX, en el trascurso de tres lustros, el barrio que se ha venido modelando conforme a la ilusión y el esfuerzo de las mujeres y hombres que lo habitan, de diversa procedencia y clase social, se consolida como el lugar saludable y privilegiado capaz de ofrecerles, a todas y a todos por igual, no sólo unas óptimas condiciones de habitabilidad frente a las entonces precarias existentes en el recinto de la población, sino también la posibilidad de involucrarse en el apasionante proyecto de construir una sociedad especialmente diversa y conciliadora donde sentirse igualmente identificadas e identificados, una sociedad avanzada capaz de posibilitar, armonizar e integrar el logro de las aspiraciones personales y laborales del amplio colectivo de individualidades y circunstancias humanas que la conforman.

Benalúa, ubicada entre dos estaciones ferroviarias, la de Madrid-ZaragozaAlicante y la de Andaluces, pronto se convirtió en el lugar de residencia de muchas familias trabajadoras del sector, en su mayoría desplazadas desde tierras castellanas, trasladadas desde otras estaciones ubicadas en las provincias de Albacete, Ciudad Real, Toledo o Madrid, y también desde Murcia, Granada o Córdoba. Estos núcleos, junto con otras familias alicantinas o de localidades cercanas a Alicante, de clase media e incluso de elevado estatus, constituyeron la primera sociedad benaluense, basada en la convivencia, en la diversidad y en el respeto como valores fundamentales (Establier Costa). Empleadas y empleados de todas las categorías y jerarquías de la escala ferroviaria (taquilleras y mozos, guardafrenos y revisores, maquinistas o fogoneros, jefes de estación o de tren, directores e ingenieros) convivieron en Benalúa junto a otros comerciantes, importantes industriales e ilustres personajes de las esferas política y cultural de la época, sumándose después otras variadas profesiones: funcionarios del cuerpo de prisiones, militares, empleadas y empleados de oficinas o comercios y un gran número de metalúrgicos, de acuerdo a las actividades e importantes servicios públicos que, como veremos, fueron integrando y conformando el barrio, enriqueciendo aún más su característica diversidad y singular modernidad (Miralles 8).

La participación de todas estas personas en la configuración de Benalúa (en su arquitectura y su urbanismo) constituyó, sin duda, la clave de su acierto (Castrechini Trotta 59-66), erigiéndose la participación ciudadana

Feminismo/s 32, diciembre 2018, pp. 231-257 
como una condición básica de la sostenibilidad del barrio (Verdaguer VianaCárdenas, «Por un urbanismo de los ciudadanos»). En la línea que proponía el diseño del barrio, versátil para adecuarse a las nuevas necesidades, los equipamientos públicos se concibieron y construyeron al tiempo que fue creciendo la ciudad y prosperaron sus habitantes. Primero, la Casa-Asilo de las Hermanitas de los Pobres (del arquitecto Guardiola, 1886), después, el proyecto de las Escuelas del barrio (Guardiola, 1889), la Iglesia de San Juan Bautista (Guardiola, 1889) y el grandioso Teatro Polo (1889). Equipamientos que, progresivamente, por su importancia llegarían a prestar servicio a Alicante, convirtiendo Benalúa en sede de relevantes instituciones administrativas y benéficas, verdaderas señas de identidad que lo caracterizarían para siempre. Así, en la flexibilidad del barrio tuvieron cabida dotaciones tan notables como la nueva Cárcel de Alicante (arquitecto González Altés, 1888) o el Cuartel de Infantería de la ciudad (ingeniero Campos Vasallo, 1900), la Estación de tranvías (1892) (entonces principal medio de comunicación con Alicante), un Mercado de abastos (1900), el Hospital del Niño Jesús (1896) y hasta un Velódromo (1896), entre otros muchos equipamientos que se irían sumando en sucesivas décadas. De este modo, el proyecto de vida en igualdad (sueño) que propone Benalúa, se materializa en la ciudad moderna sostenible, en la ciudad equipada que permite tanto el funcionamiento de los servicios públicos más específicos como el desarrollo de las actividades humanas más cotidianas (Fig. 3).

El significado integrador y conciliador de la propuesta, favoreciendo la participación de todas sus ciudadanas y ciudadanos, se acentúa y constata en el logro real de una permeabilidad eficiente entre los ámbitos privado y público (Valera Pertegàs 22-24). Rompiendo con el planteamiento neoclásico, los espacios públicos y privados se suceden sin jerarquía en la retícula planificada, en la misma escala humana de lo propio, igualmente accesibles, garantizando los variados flujos de circulación posibles y el desarrollo de las diversas funciones y roles sociales de mujeres y hombres. El orden de la malla ortogonal seriada, tan estricto como flexible, permite esta transición de forma dinámica, natural y homogénea. La manzana de 100x40 metros cuadrados constituye la matriz, la unidad válida tanto para la construcción de viviendas (manzanas residenciales) como de equipamientos o plazas (manzanas dotacionales).

Feminismo/s 32, diciembre 2018, pp. 231-257 
Integración de la perspectiva de género en el estudio de la ciudad y su patrimonio: aprendiendo de la experiencia de Benalúa hacia un futuro más sostenible

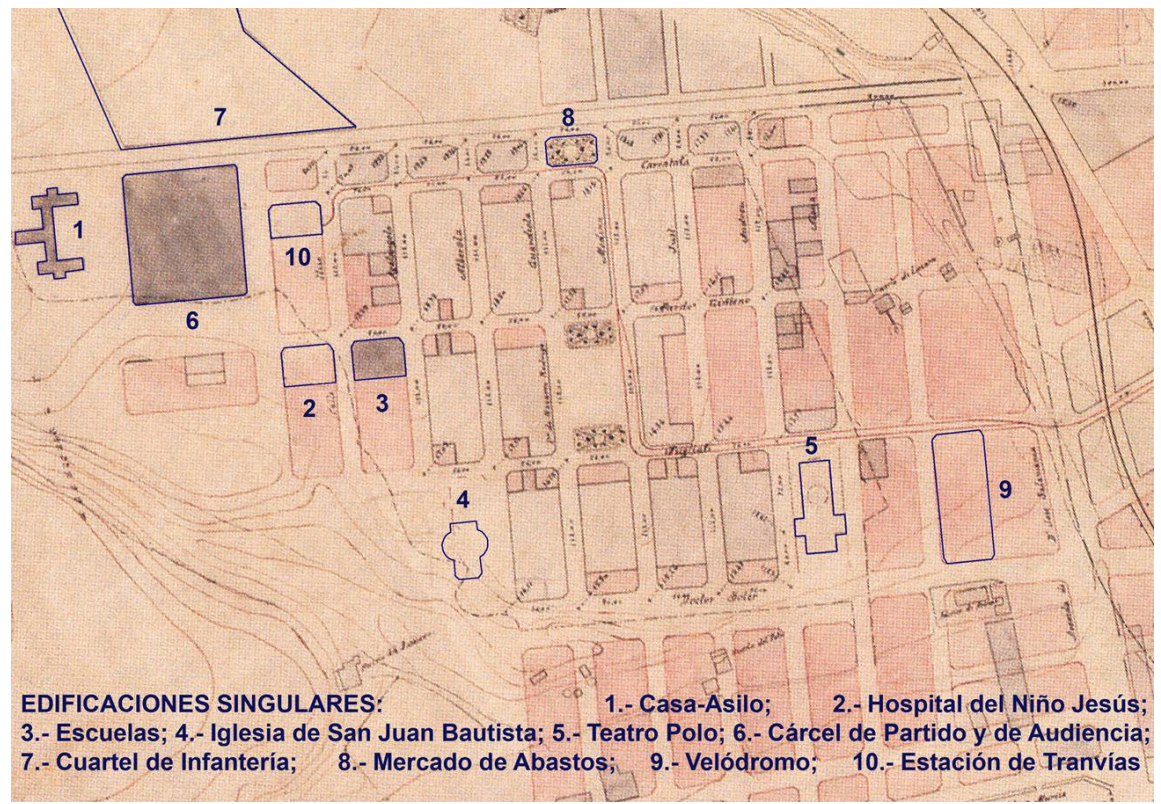

Figura 3. Edificios singulares en Benalúa que comenzaron su construcción o gestión antes de finalizar el siglo XIX. Se representan sobre el «Plano de Alicante 1897». Fuente: elaboración propia.

De este modo, las arquitecturas singulares, al igual que las domésticas, pasan a ocupar una de estas manzanas (cualquiera) dentro de la trama previamente concebida, eliminando privilegios fronterizos o restricciones de acceso que pudieran mermar la movilidad potencial entre los diferentes espacios. Como centro neurálgico, también la plaza abierta ajardinada se ajusta a esta modulación, al orden lógico que, lejos de condicionar o restringir los comportamientos humanos, confiere idéntica categoría a todas las funciones y relaciones (públicas y privadas) que conforman la ciudad. Y es precisamente esta plaza, intersección de todos los flujos de movimiento, con libertad absoluta de acceso y de tránsito, la que intencionadamente, con mayor relevancia que cualquier edificación, alienta a la participación ciudadana constituyendo el centro público del barrio (Calduch Cervera y Varela Botella 51-55).

Feminismo/s 32, diciembre 2018, pp. 231-257 
Integración de la perspectiva de género en el estudio de la ciudad y su patrimonio: aprendiendo de la experiencia de Benalúa hacia un futuro más sostenible

\section{LA CASA BENALUENSE, ENTRE LO PÚBLICO Y LO PRIVADO}

Para nosotros lo público y lo privado son dos polos; entre uno y otro extremo, reconocemos una serie de espacios intermedios. Lo que nosotros vemos son transiciones. $Y$ en esto estamos de acuerdo con el urbanismo, que considera que son esencialmente estas transiciones entre estos polos las que constituyen la particular calidad de las secuencias espaciales de la ciudad (Schlack 25).

Probablemente, Benalúa sea de los primeros casos en la ciudad de Alicante donde encontremos un proyecto completo del edificio (Fig. 4), con sus plantas, alzados y detalles constructivos, anticipándose en el estudio del problema de la vivienda para proponer soluciones a las precarias condiciones higiénicas de la época (Bevià i Garcia y Varela Botella 103-106). Este hecho nos anuncia la importancia vital que la vivienda va a tener en la formación del barrio.

El diseño de la casa, de cuyo cuidado socialmente se responsabiliza a la mujer, adquiere en el barrio especial protagonismo. Son los propietarios, de acuerdo a los tipos de edificación que precisan, los que determinan la morfología urbana (Calduch Cervera, La Ciudad Nueva), suponiendo un importante avance en la aplicación de los principios higienistas con respecto a las primeras planificaciones humildes desarrolladas en la ciudad (mayores superficies, patios abiertos, adecuada orientación, iluminación y ventilación). De este modo, la forma urbana y los tipos edificatorios constituyen una misma unidad (Alonso Salvador, Blasco Sánchez y Piñón Pallarés 66-69). Viviendas,

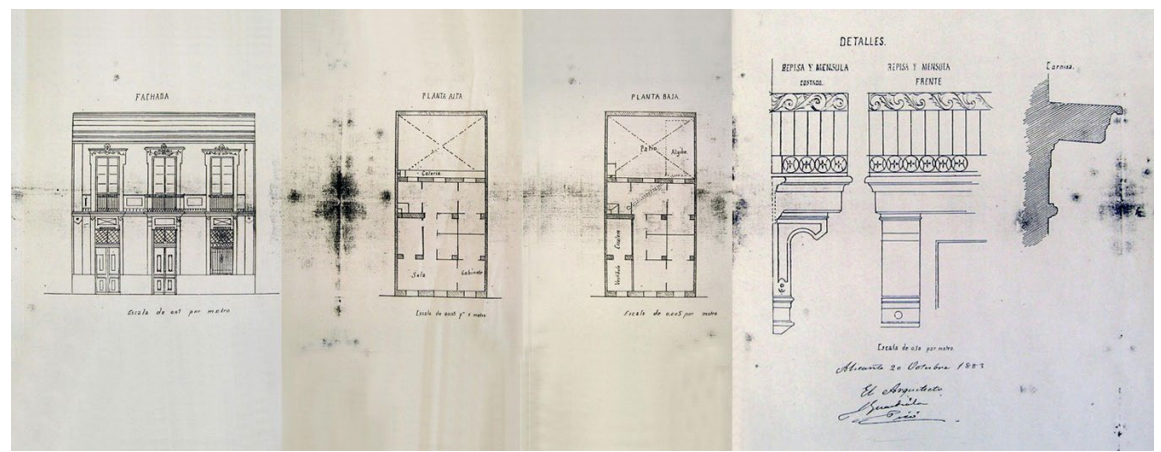

Figura 4. Plano de alzado, plantas y detalles constructivos de una casa (arquitecto Guardiola Picó, 1883). Fuente: Archivo Histórico Municipal de Alicante.

Feminismo/s 32, diciembre 2018, pp. 231-257 
parcelas y manzanas, forman parte de un todo coherente integrado en la retícula, planteando, desde su propia concreción, el discurso recurrente de las secuencias espaciales que conforman la ciudad, transición subjetiva entre los espacios públicos y privados. La vivienda en Benalúa no es un cuerpo aislado o cerrado, es la célula privada, en la medida en que cada propietaria o propietario guarda, de la que surge el mismo entorno compartido, la ciudad pública, favoreciendo la transversalidad de las funciones que dentro y fuera de la casa se desarrollan.

Partiendo desde este posicionamiento: la vivienda como principio y protagonista urbano, si bien la casa benaluense se proyecta en un momento en que la mayoría de las mujeres se dedican casi en exclusividad al hogar y a la familia, también atiende en su diseño a las relaciones que establece con el resto de las viviendas (que se conciben igualmente orientadas y equipadas) y el medio ciudadano (espacios de transición, control del soleamiento, recorridos urbanos), permitiendo conciliar funciones y responsabilidades personales y laborales, así como los tiempos de ocio. Para lo cual, considerando la diversidad familiar y sus particulares necesidades en cada etapa de la vida, la participación de las personas que van a vivir en ellas resulta fundamental.

Benalúa, respondiendo a su pluralidad consustancial, propone una doble estrategia de residencia que integra, por un lado, un modelo de casa suficientemente amplio y versátil para poder adecuarse al perfil familiar en todas y cada una de sus etapas, al alcance de las clases medias trabajadoras, y, por otro, la venta de solares para ser edificados directamente por iniciativa particular, obviamente accesibles para un sector más favorecido de la población; compartiendo los mismos equipamientos básicos y servicios urbanos financiados proporcionalmente. Ambas opciones implican adquirir una misma categoría, la de propietarios de una casa, cuando lo habitual en el momento, para las clases menos pudientes, era habitarlas en régimen de alquiler.

El modelo de casa de 10x20 metros cuadrados, unidad a la que tiene acceso una familia trabajadora, se compone de dos plantas, baja y piso, acondicionadas en principio para alojar dos viviendas, de aproximadamente 120 metros cuadrados cada una, con entradas independientes desde la calle. La relación directa de ambas plantas con la vía pública favorecerá en muchos sentidos las relaciones personales y comerciales en el barrio que, hasta bien 
entrado el siglo XX, convivirá a puertas abiertas, sin ser ésta una posibilidad exclusiva de los bajos.

Es un buen punto de partida considerar que, en base al diseño de la casa y según las necesidades familiares en un determinado momento, puede hacerse más apta una de las plantas para habitar, cualquiera de ellas, e independientemente disponer de la segunda para el desarrollo de cualquier otra actividad, tanto de la misma familia como totalmente ajena.

En un primer nivel en que se precisa el conjunto de la casa como alojamiento, frecuente en el momento por tener que convivir (por dificultades económicas) varias hermanas o hermanos con sus respectivas familias en la vivienda de los progenitores, el modelo palía sustancialmente la carencia de intimidad y favorece obviamente la convivencia, situación que especialmente les incomoda a ellas, quienes deben permanecer más tiempo al cuidado de la casa, sin ni siquiera sentirla propia.

En un segundo nivel más relajado, podría iniciarse una actividad económica. Bien alquilando a terceros como alojamiento o lugar de trabajo, bien implicándose la misma familia. De este modo, el esfuerzo realizado en adquirir una casa no revierte exclusivamente en lograr un espacio propio donde residir, abre además un abanico de posibilidades al desarrollo y la economía familiar, como extensión de la propia vivienda y de la propia vida. Iniciativas en las que las mujeres, especialmente, encontrarán la oportunidad de incorporarse al mundo productivo frente al tradicional reproductivo, sin desatender sus responsabilidades familiares y domésticas.

Como vemos y esto es lo notable, mucho antes de las propuestas de conjuntos de viviendas que revisan la Modernidad (véase el PREVI de Lima; García-Huidobro, Torres Torriti, y Tugas) en el sentido de entender la casa no como un bien de consumo (un producto acabado, definido, cerrado, pasivo) sino como un bien de producción (abierto, activo, flexible, dinámico), Benalúa ya la concibe como un proceso en cuyos crecimientos y transformaciones sus habitantes lideran las acciones al ritmo que demandan sus vidas. $\mathrm{Y}$, en estas iniciativas, las mujeres tienen un papel relevante porque, entre otras razones, la casa deja de ser para ellas lugar de encierro para devenir oportunidad de relación.

Y, además, con posibilidades de éxito, porque la ciudad que surge requiere equiparse y autoabastecerse. Así, gran parte de las plantas bajas comenzaron

Feminismo/s 32, diciembre 2018, pp. 231-257 
tempranamente a funcionar como pequeñas empresas o comercios, al ritmo que imprimía el natural crecimiento del barrio. Por el modo en que influyeron en su desarrollo, son tres las conclusiones (cualidades) que, a priori, deben destacarse: en primer lugar, la participación ciudadana se consolida y muestra imprescindible para entender el acierto de Benalúa. Son las mujeres y los hombres, primeros benaluenses, quienes con su esfuerzo activo (especialización) logran atender las demandas de una sociedad en constante desarrollo. En segundo lugar, las empresas familiares, actividades comerciales o servicios profesionales que espontáneamente surgen como extensión de la vivienda, funcionan además como espacios de transición entre las esferas de lo público y lo privado, espacios que encajan perfectamente en el diseño de la casa benaluense. Y, por último, la integración de estos comercios en las casas garantiza el reparto dinámico, la distribución y acceso de todas y todos por igual a los equipamientos básicos, en definitiva, garantiza el funcionamiento del barrio y el bienestar de una sociedad, conciliando en equilibrio los tiempos y espacios que se requieren para la vida (para el trabajo, el descanso y la dedicación familiar).

Un último aspecto a tener en cuenta se centra en la propuesta de distribución de la vivienda (Fig. 5), aunque ésta siempre es susceptible de ser modificada, pudiendo variarse la tabiquería interior dependiendo de las necesidades en cada momento. La casa de Benalúa emplea el sistema combinado de muros de carga (en el perímetro) y pilares intermedios, a pesar de que en el momento solía construirse la totalidad de la estructura a base de muros solamente (por la menor dimensión de fachada). El único elemento fijo es la caja de escalera (iluminada por claraboyas dispuestas en el tejado) que se aprovecha para agrupar las zonas húmedas de la vivienda, las que requieren de instalaciones (cocina y retrete exterior), quedando dos de los tres vanos en que se desarrolla el programa totalmente libres, a disposición de su propietaria o propietario.

Si bien el modelo de Benalúa responde al tipo consolidado en las áreas planificadas de carácter humilde incorporadas a la ciudad en la primera mitad del siglo XIX, incluye variaciones claras para conseguir mejorar sus condiciones higiénicas. Sin duda, la más evidente se refiere a la dimensión de los patios (al aumentar la superficie de la parcela). La vivienda se construye con un fondo aproximado de trece metros, disponiendo patios posteriores con

Feminismo/s 32, diciembre 2018, pp. 231-257 


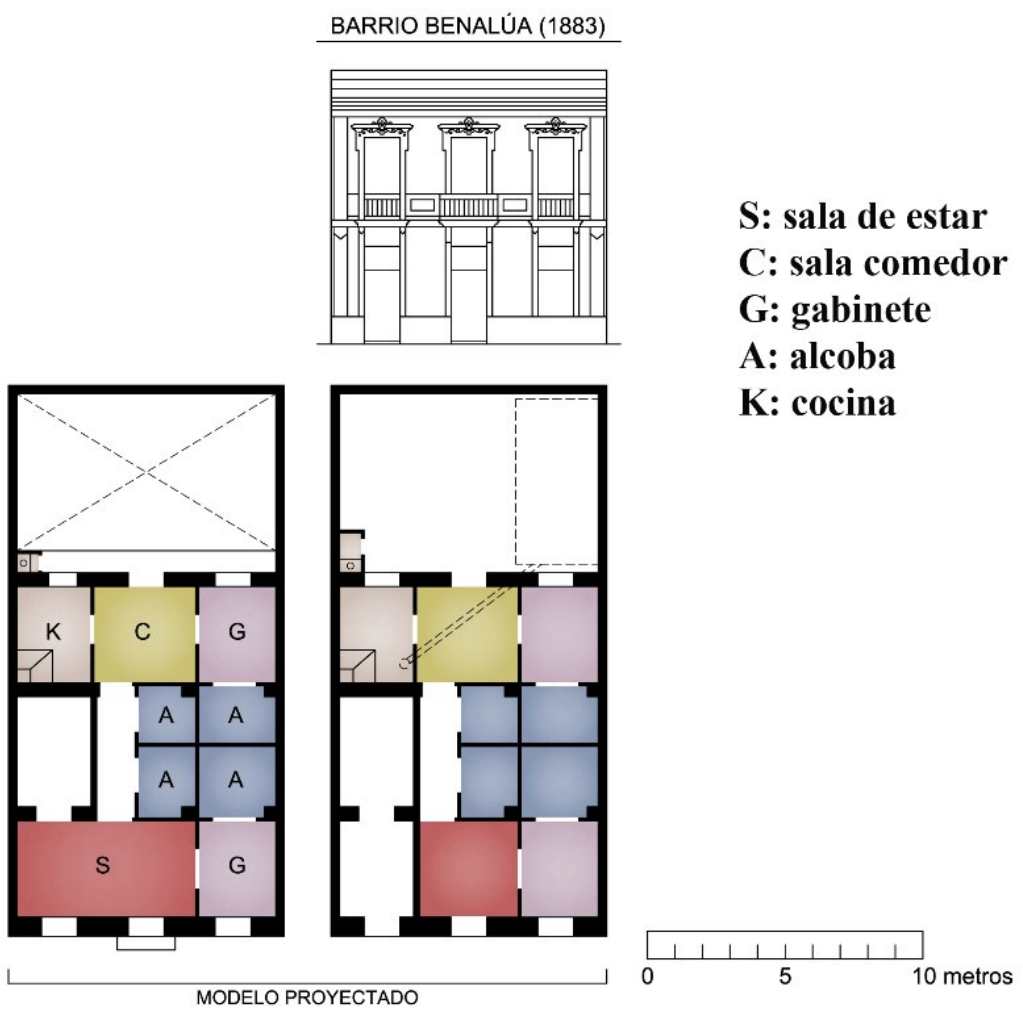

Figura 5. Módulo de una casa para dos viviendas con accesos independientes desde la calle. Fuente: elaboración propia.

una profundidad aproximada de siete, más de un tercio de la dimensión del solar, resultando una superficie cuatro veces mayor que la mínima exigida por la normativa de la época.

De este modo, la vivienda entre medianeras se concibe realmente con dos fachadas (de diez metros), una exterior a la calle y otra interior al generoso patio, separadas por un fondo edificable que permite garantizar la iluminación y ventilación cruzada de todo el alojamiento (de Este a Oeste). Aunque, según el modo de proceder de la época, todavía algunas estancias (alcobas) se disponen interiores.

Feminismo/s 32, diciembre 2018, pp. 231-257 
El programa se distribuye de forma simétrica respecto a ambas fachadas, recibiendo éstas el mismo tratamiento, cada una cumple su cometido y es imprescindible en el funcionamiento conjunto de la casa. No se conciben espacios de primera o segunda categoría, servidos o sirvientes, relegados (residuales), con igual significación se integran en la vivienda, favoreciendo la armonía de todos los usos, más o menos privados o públicos, que se desarrollan en el marco del hogar. De este modo, el mismo orden que caracteriza el trazado urbano, sin jerarquías, se percibe ahora en el diseño de la casa, su núcleo más íntimo y personal en el que las mujeres y los hombres también conviven (se desarrollan y educan) en igualdad.

En la misma escala, presencia y dimensión, se disponen, con igual categoría, en cada una de las fachadas, las piezas que articulan el funcionamiento activo de la casa, los espacios que garantizan que los diferentes usos cotidianos, reconociendo a todos su importancia, se desarrollen en equilibrio a lo largo del día. Preparar los alimentos o preocuparse de la limpieza doméstica, comer o cuidar de la higiene personal, adquieren en el diseño la misma relevancia que el resto de las relaciones sociales que, como transición a las públicas, se realizan dentro de la casa. La sala de estar, por la que se produce el acceso a la vivienda, la sala comedor en relación con la cocina, donde la familia se reúne con mayor privacidad, el desahogado patio, los gabinetes y las alcobas, se organizan en la secuencia lógica y dinámica que significa adentrarse en la intimidad del hogar, sin perder la calidad y dignidad que merecen cada uno de los espacios que, en definitiva, componen y concilian el escenario de la vida familiar.

\section{PATRIMONIO MATERIAL E INMATERIAL: EL LEGADO DE UNA SOCIEDAD MODERNA}

El patrimonio no es sinónimo de monumentos y objetos sin vida, arquitectónicos, artísticos o expuestos en un museo. El patrimonio es la identidad cultural de una comunidad y es uno de los ingredientes que puede generar desarrollo en un territorio, permitiendo equilibro y cohesión social (Molano 76).

Definir el patrimonio como construcción social (Prats) es la tesis avanzada que fundamenta, a priori, el necesario conocimiento de las sociedades,

Feminismo/s 32, diciembre 2018, pp. 231-257 
potencialmente creativas y constructoras, para poder reconocer su aportación patrimonial. Un conocimiento parcial, infravalorando las diferencias propias de una sociedad diversa, supone por tanto negar el patrimonio, renunciar a la memoria que nos identifica y en la que nos reconocemos (somos), tergiversar la historia silenciando sentidos y contenidos, valores esenciales de igualdad, equilibrio y cohesión social (Molano 69-84). Obviar la perspectiva de género como parámetro crucial en la valoración patrimonial conlleva, en consecuencia, la descontextualización del patrimonio, tanto material como inmaterial, el desconocimiento absoluto de su significado y, por ende, la pérdida insensata de la identidad que determina el futuro de una sociedad.

Fue el sentido de hermandad benaluense, integrador de diferentes grupos sociales (profesionales, empresarios, comerciantes o trabajadores), el que propició, desde su nacimiento, una intensa actividad social y cultural en el barrio. Y fueron sus propias gentes, entre las que se encontraban muchos de los ilustres personajes de la época, conviviendo y poniendo en valor sus diferencias, las que terminaron consolidando su singular modernidad: Juan Miró Moltó, Trino Esplá Visconti, Francisco Figueras Bushell, y sus hijos, Gabriel Miró Ferrer, Oscar Esplá Triay, Francisco Figueras Pacheco, entre otros muchos defensores del patrimonio educativo y la cultura universal, residieron en Benalúa.

Entre el amor por la cultura y las manifestaciones artísticas se formaron las primeras generaciones benaluenses, rodeadas de un sinfín de hogares comunes. Con claras intenciones progresistas, se instaló tempranamente en la plaza el Casino La Unión (1898), convirtiéndose en centro de reunión donde desarrollar tertulias, juegos, conferencias, sobre toda clase de materia, ciencia o nueva teoría del momento, además de otros locales como el Ramón de Campoamor, el Salón Senabre (conocido como Ateneo Senabrino), las aulas del maestro Flores Castillo o La Peña (que incorporó al barrio una nutrida biblioteca) (Martínez Mena 79-118).

No debe creerse, por tanto, casual, el modo en que el barrio decidió afrontar la educación de las primeras generaciones nacidas en Benalúa. La convicción y el empeño en la necesidad primordial de procurar la educación a todas las niñas y a todos los niños sin distinción, merece destacarse como una de sus principales conquistas. Posibilitar a las mujeres adquirir en igualdad la formación que abriría las puertas a sus aspiraciones personales y

Feminismo/s 32, diciembre 2018, pp. 231-257 
laborales, reconociendo la cultura como patrimonio colectivo, forma parte del proyecto de vida que propone Benalúa, en el último tercio del siglo XIX y en una ciudad española de provincias. De este modo, la educación, la información y la participación, como forma de vida en el barrio, se convierten en los pilares que hacen posible de la sociedad benaluense una sociedad más sostenible (Fariña Tojo 25).

Será de nuevo la casa, como extensión de la vivienda, la que permitirá desarrollar las labores de enseñanza y aprendizaje en las etapas tempranas, previas a la construcción de un edificio propio de escuelas. Frente a la carencia de centros públicos organizados, varias viviendas del barrio fueron habilitadas como aulas docentes, transformándose en locales totalmente diáfanos, equipados con bancos y pupitres de madera, planos y pizarras. Benalúa disponía a principios de siglo de tres escuelas, una de ellas mixta. Se ubicaba ésta última en la misma plaza central del barrio, enfrentada al Casino. En el primer piso, se encontraba la escuela de niñas de doña Carmen Albert y, en la planta baja, atendía a los niños el maestro Flores Castillo, compartiendo la casa como espacio integrador y la plaza como lugar común de recreo, adquiriendo en igualdad los saberes y los juegos que, desde la base, determinan los futuros potenciales y oportunidades de mujeres y hombres.

En el empeño de Carmen Albert, continuaron Finita y María de los Ángeles Carratalá Figueras, impartiendo las clases en el Aula Primaria establecida en su propia casa y, a partir de 1920, la Comunidad de las Hijas de María Auxiliadora (Salesianas de Don Bosco), que edificaron su Colegio-Convento en el barrio. A comienzos de la década de los treinta, en el mismo recibían clases gratuitas un total de doscientas veinticinco niñas durante el día y un grupo de ochenta muchachas obreras durante la noche. Sin duda, tratar de paliar, a escala de barrio, las dificultades de las mujeres para acceder a la educación (Ballarín Domingo 245-260) y enorgullecerse de ello (porque todas las niñas benaluenses adquirían realmente una educación básica cuando, cuanto menos, no se consideraba necesario), supone uno de los logros más ambiciosos y actuales que deben reconocerse como patrimonio cultural de Benalúa.

La mujer benaluense no es, por tanto, una mujer callada o silenciada: es, por el contrario, una mujer activa que se implica constantemente en la lucha social. Numerosas actuaciones constatan su presencia e influencia en el hecho arquitectónico y urbanístico, por ejemplo. En definitiva, es del rol

Feminismo/s 32, diciembre 2018, pp. 231-257 
social femenino del que, de forma evidente, se nutre y equilibra la ciudad, determinando su configuración y valor patrimonial. En un fragmento de ciudad de nueva planta, segregado de la ciudad convencional y de sus convenciones, las mujeres, convincentes y convencidas, se sienten libres y seguras para tomar las riendas de sus decisiones, al margen de prejuicios. Y su papel en la construcción de esa sociedad nueva resulta relevante. Y, hasta el presente trabajo, desconocido, ignorado y silenciado (parafraseando a Calduch Cervera, 99 Adis).

Y no sólo nos referimos al patrimonio inmaterial, si bien hasta el momento pueden haberse seguido caminos que no han dejado rastro material en las investigaciones sobre patrimonio arquitectónico y urbano (Huguet Santos 255-261), la perspectiva innovadora de género, y así se reivindica en este artículo, es capaz de desvelar y actualizar, en estas disciplinas, multitud de contenidos y discursos cruciales en la no negación del patrimonio.

La energía vital de las mujeres, siempre receptivas, ha impulsado el desarrollo de intervenciones determinantes en el contexto urbano, especialmente en su patrimonio. Ellas, brillantemente organizadas, con claros objetivos comunes, aprovechando sus diferencias, como promotoras desde los estatus más privilegiados o como trabajadoras responsables desde las clases más humildes, han forjado ideas, concebido planes eficaces de actuación y culminado las iniciativas, siempre en el empeño y ejercicio de su compromiso social.

En definitiva, proponemos, tomando como ejemplo paradigmático el barrio de Benalúa y su arquitectura, incorporar a los indicadores de valoración del patrimonio, en virtud de los cuales éste se protege en mayor o menor grado, las aportaciones que nos proporciona la perspectiva de género. Tradicionalmente, estos indicadores toman en consideración aspectos que atienden a la antigüedad (una perspectiva arqueológica), a la forma (oscilando entre el estudio tipológico y morfológico, disciplinar, y las catalogaciones estilísticas, preferidas por los historiadores), a la función (si el edificio está o no en uso) y a la técnica (innovaciones o aportaciones en materiales, soluciones constructivas, etc.), pero no toman nota de valores tales como los espacios públicos de relación, los equipamientos y servicios, la movilidad y accesibilidad, las viviendas, la seguridad y la participación ciudadana (Muxí Martínez et al. 105-130), variables todas ellas pertinentes no sólo en el análisis y en el

Feminismo/s 32, diciembre 2018, pp. 231-257 
proyecto de la ciudad y su arquitectura, sino también y además en su puesta en valor como patrimonio sostenible de todos. Benalúa demuestra resplandecientemente que su valor reside, precisamente, en la calidad de todos y cada uno de estos niveles de la ciudad y la casa.

\section{ALGUNOS APUNTES PARA EL SUMA Y SIGUE...}

A modo de epílogo (que lejos de concluir avive el debate), describiremos brevemente dos ejemplos:

- (1885): Enriqueta Waring y Hernández de Tejada y su esposo (si bien únicamente suele citarse a éste) ceden terrenos a la Congregación de las Hermanitas de los Pobres para que edifiquen un asilo en Benalúa y financian su construcción (Pardo Jimeno). Con total responsabilidad, las hermanas llevan a cabo la obra y el trabajo diario en la Casa-Asilo, mantienen y reparan el inmueble, labor que continúan ellas, las Hijas de la Caridad de San Vicente de Paúl. Gracias al esfuerzo y cuidado de las mujeres que habitaron esta Casa, surge un valioso regalo cargado de historia para la ciudad de Alicante, la magnífica e imponente Capilla de finales del XIX que todavía hoy alberga, enriqueciendo con creces nuestro patrimonio (actualmente en espera de demolición).

- (1896): Victorina Amérigo de Garriga cede terrenos para la construcción de un hospital infantil en Benalúa. El Hospital del Niño Jesús (Fig. 6), propiedad de la Junta de Damas constituida al efecto, liderada por Victorina Amérigo, funciona en 1906 como asilo nocturno para mujeres sin hogar. La construcción (decepción) pasa a manos del Ministerio de la Guerra y es demolida en 2007 ¿Nadie se preguntó por qué la Comandancia Militar de Ingenieros respondía a la tipología de espina de pez, con un cuerpo central longitudinal de dos alturas (para las zonas comunes: despachos, consultas, quirófanos) y dos naves laterales de una sola planta (para albergar las camas, a los enfermos)? Alarmante resulta esta pérdida para el patrimonio alicantino, símbolo de la concienciación y aplicación más avanzada de los principios higienistas y del desarrollo que en el sistema hospitalario local, en la transición de los siglos XIX al XX, impulsaron decididos colectivos de mujeres.

Feminismo/s 32, diciembre 2018, pp. 231-257 
Integración de la perspectiva de género en el estudio de la ciudad y su patrimonio: aprendiendo de la experiencia de Benalúa hacia un futuro más sostenible

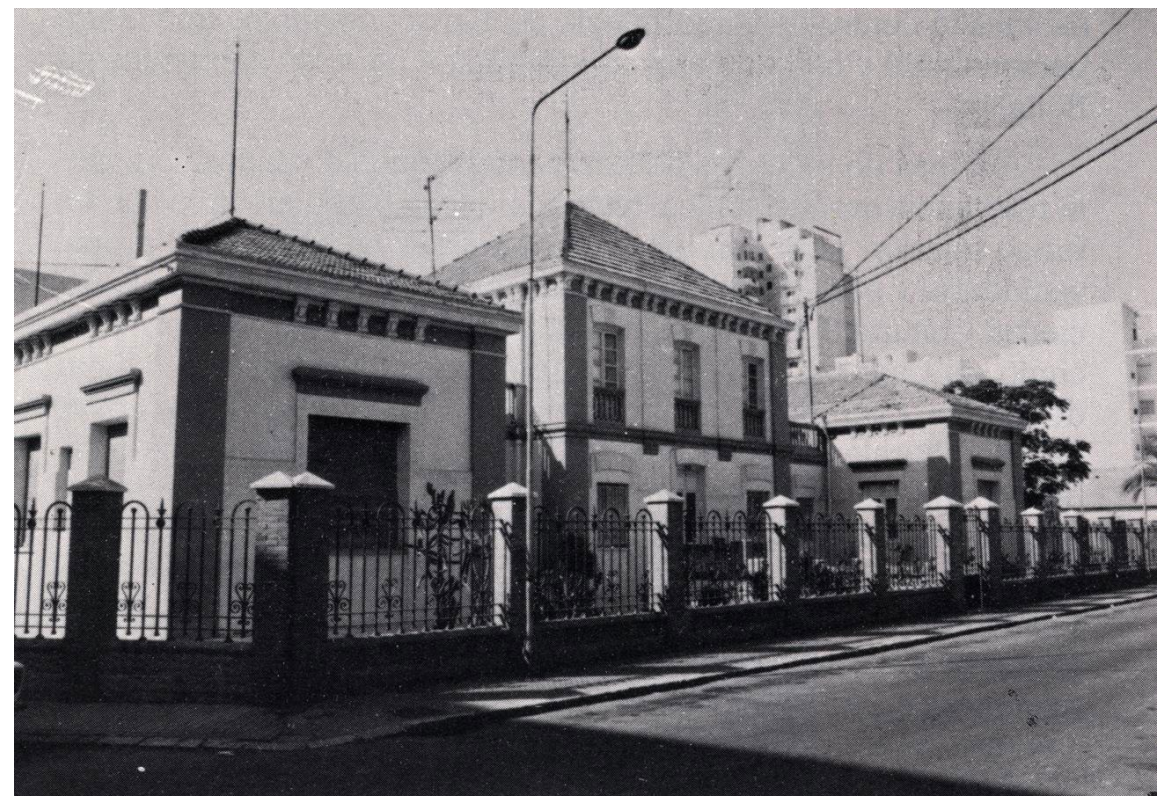

Figura 6. Hospital del Niño Jesús, en los años ochenta del siglo XX. Demolido en 2007. Fuente: (Andrés Carratalá 88).

\section{CONCLUSIONES}

Ante el reto que nos plantea la Agenda 2030 para el Desarrollo Sostenible: la creación de un nuevo modelo a través de la consecución de 17 Objetivos de Desarrollo Sostenible y, concretamente en nuestro caso, «Objetivo 11. Lograr que las ciudades y los asentamientos humanos sean inclusivos, seguros, resilientes y sostenibles», se hace necesario identificar y aprender de las buenas prácticas que verdaderamente han logrado mejorar la calidad de vida de las personas.

Benalúa es uno de esos casos que, como indica Hernández Aja, puede calificarse de buena práctica. En primer lugar, por proporcionar unas condiciones para la vida urbana muy mejoradas y deseables. En segundo lugar, por entender los conceptos de planificación y gestión urbana de una manera más participativa e inclusiva, favoreciendo el sentido de comunidad. En tercer lugar, por representar un modelo sostenible, social, cultural, económica y

Feminismo/s 32, diciembre 2018, pp. 231-257 
ambientalmente. Y, por último, por defender un modelo socialmente inclusivo, independientemente del género, etnia, cultura o economía, en su complejo contexto específico.

De la investigación se concluye que valores tales como los espacios públicos de relación, los equipamientos y servicios, la movilidad y accesibilidad, las viviendas, la seguridad y la participación ciudadana, son variables pertinentes no sólo en el análisis y en el proyecto de la ciudad y su arquitectura, sino también y muy especialmente en su puesta en valor como patrimonio, actuando éste como impulso del desarrollo sostenible. Por ello, tomando como ejemplo el barrio de Benalúa, en este artículo se propone incorporar a los indicadores de valoración del patrimonio sostenible, las aportaciones que nos proporciona la perspectiva de género.

\section{REFERENCIAS BIBLIOGRÁFICAS}

Alonso Salvador, Matilde, M. Carmen Blasco Sánchez y Juan Luis Piñón Pallarés.

$V$ Siglos de Arquitectura. Alicante: Colegio Oficial de Arquitectos de la

Comunidad Valenciana, Demarcación de Alicante, 1990.

Andrés Carratalá, M. Asunción. El barrio de Benalúa en Alicante: estudio de geografía urbana. Alicante: Publicaciones de la Caja de Ahorros Provincial de Alicante, 1989.

Ballarín Domingo, Pilar. «La educación de la mujer española en el siglo XIX». Historia de la Educación: Revista interuniversitaria 8 (1989): 245-260.

Bevià i Garcia, Màrius y Santiago Varela Botella. Alicante: Ciudad y Arquitectura. Alicante: Fundación Cultural CAM, 1994.

Calduch Cervera, Joan. La Ciudad Nueva. La construcción de la ciudad de Alacant en la primera mitad del siglo XIX. Alicante: Patronato Municipal del Quinto Centenario de la Ciudad de Alicante, 1990.

Calduch Cervera, Joan. 99 Adis: diccionario de arquitectos desconocidos, ignorados y silenciados. Valencia: Colegio Oficial de Arquitectos de la Comunidad Valenciana, 2004.

Calduch Cervera, Joan y Santiago Varela Botella. Guía de Arquitectura de Alacant (1. ${ }^{\circ}$ Tomo). Alicante: Colegio de Arquitectos de Alicante, 1979.

Castrechini Trotta, Angela. «Retos de la psicología ambiental en el diseño urbano participativo». Psicología de la ciudad, debate sobre el espacio urbano. Barcelona: Editorial UOC, 2008. 59-66.

Feminismo/s 32, diciembre 2018, pp. 231-257 
Integración de la perspectiva de género en el estudio de la ciudad y su patrimonio: aprendiendo de la experiencia de Benalúa hacia un futuro más sostenible

Col-lectiu Punt 6. «Construyendo entornos seguros desde la perspectiva de género». Programa Ciutats i Persones, Institut de Ciències Politiques i Socials. Informe número 5, 2011.

Echarri, María de. «Crónica del movimiento católico femenino». Revista Católica de cuestiones sociales. 234 (1914): 434-437.

Establier Costa, Rafael. Añoranzas y recuerdos benaluenses. Alicante: Publicaciones de la Caja de Ahorros Provincial de la Excma. Diputación de Alicante, Editorial Confederación Española de Cajas de Ahorros, 1976.

Fariña, José. «Sostenibilidad y racionalidad de los procesos de urbanización». Boletín CF+S 24. Ecología y ciudad: raices de nuestros males y modos de tratarlos (2014): 25-28.

García-Huidobro, Fernando, Diego Torres Torriti y Nicolás Tugas. ¡El tiempo construye! Barcelona: Gustavo Gili, 2008.

Giménez García, Efigenio, Jaume Giner Álvarez y Santiago Varela Botella. Sobre la ciudad dibujada de Alicante, del plano geométrico al plan general de 1970. Alicante: Colegio Oficial de Arquitectos de Valencia, Delegación de Alicante, 1985.

Gutiérrez Lloret, Rosa Ana. «La época de la Restauración». Historia de Alicante (Tomo II). Alicante: Ayuntamiento de Alicante, Patronato del Quinto Centenario de la Ciudad de Alicante, 1990. 541-560.

Hernández Aja, Agustín. «Informe Sobre la Evolución de las Buenas Prácticas Españolas y su Relación con el Cumplimiento del Programa Hábitat». Ciudades para un futuro más sostenible. ETSAM: Madrid, 2001. 17 mayo 2018. $<$ http://habitat.aq.upm.es/evbpes/abpes_2.html\#9>

Hernández Aja, Agustín, Isabela Velázquez Valoria, y Carlos Verdaguer VianaCárdenas. «Ecobarrios para ciudades mejores». Ciudad y Territorio Estudios Territoriales 41.161-16 (2009): 543-558.

Huguet Santos, Montserrat. «El género de la Memoria». THÉMATA - Revista de Filosofía 39 (2007): 255-261.

Martínez Mena, Manuel. «Centenario, pincelada cultural. Benalúa en Alicante, Alicante en Benalúa». I Centenario del barrio de Benalúa. Alicante: Banco de Alicante, Grupo Banco Exterior, 1985. 79-118.

Miralles, Virgilio. «Benalúa, el barrio libertado». Diario Información 14 octubre 1956: 8.

Molano, Olga Lucía. «Identidad cultural un concepto que evoluciona». Revista Opera 7 (2007-2008): 69-84.

Feminismo/s 32, diciembre 2018, pp. 231-257 
Muxí Martínez, Zaida et al. «¿Qué aporta la perspectiva de género al urbanismo?». Feminismo/s 17 (2010): 105-130.

Nash, Mary. Mujer, familia y trabajo en España, 1875-1936. Barcelona: Anthropos, 1983.

ONU. Asamblea General. Transformar nuestro mundo: la agenda 2030 para el Desarrollo Sostenible. 2015. 17 mayo 2018. <http://www.un.org/es/comun/ docs/?symbol=A/RES/70/1>

Pardo Jimeno, Pascual. Memoria escrita y leída por don Pascual Pardo y Jimeno, iniciador de la Sociedad anónima Los Diez Amigos, en la tercera Junta General de accionistas celebrada el 17 de enero de 1886. Alicante: Imprenta de Juan José Carratalá, 1886.

Ponce Herrero, Gabino y Juan Manuel Dávila Linares. «Medidas higienistas y planes de reforma urbana en el tránsito de los siglos XIX al XX en las principales ciudades de la provincia de Alicante». Investigaciones Geográficas 20 (1998): 141-159.

Prats, Llorenç. Antropología y patrimonio. Barcelona: Editorial Ariel S. A., 2009. Schlack, Elke. «Espacio público». ARQ 65 (2007): 25-27.

Valera Pertegàs, Sergi. «Espacio privado, espacio público: dialécticas urbanas y construcción de significados». Tres al Cuarto 6 (1999): 22-24.

Velázquez Valoria, Isabela. «El tiempo de las cerezas. Reflexiones sobre la ciudad desde el feminismo». Cuadernos de Investigación Urbanística 42 (2004): 67-74.

Verdaguer Viana-Cárdenas, Carlos. «Por un urbanismo de los ciudadanos». Ecología y ciudad: raíces de nuestros males y modos de tratarlos. Madrid: Editorial El Viejo Topo, 2003. 1-15.

Verdaguer Viana-Cárdenas, Carlos. «El campo y la ciudad, áreas de reencuentro. Hacia una Nueva Cultura del Territorio». Hábitat y Sociedad 6 (2013): 11-40.

Feminismo/s 32, diciembre 2018, pp. 231-257 